\title{
La información como industria
}

Saúl Armendáriz Sánchez*

\begin{abstract}
RESUMEN
La información analizada como una industria, es el tema principal de este artículo, en primer lugar, se presenta un panorama general de la economía de la información, y se resalta el poder político, social y cultural de ésta. Posteriormente se exponen de manera amplia diversos tópicos de la información como industria, así como los factores que intervienen para su funcionamiento y desarrollo. En un tercer punto, se lleva a cabo un estudio de lo que es la información como industria en México, donde se distingue a la información lucrativa de la no lucrativa, al igual que la información científico-técnica y su consulta por medio de bases de datos.
\end{abstract}

Para finalizar, se examina la educación bibliotecológica nacional y su relación con esta industria.

\section{ABSTRACT}

In this paper an análisis is made of information as an industry. Firstly, an overview of the economics of information is presented and its political social and cultural power is underlined. Then several related topics are presented in a broad manner, as well as the factors which come into play in order for information as an industry to be able to function and develop.

A third point deals with a study of information as an industry in Mexico and a distinction is made between information for profit and that which does not involve profit. Technical/scientific information is considered as well as the way people consult this type of information using data banks.

\section{LA ECONOMÍA DE LA INFORMACIÓN}

T a información es, y ha sido y será una de las partes fundamentales en el desarrollo tecnológico, económico, científico, cultural, social y político de cualquier país, por la amplia gama de posibilidades de uso que ofrece, así como por su contenido propio.

La información es producida por distintas fuentes que persiguen objetivos diferentes, casi todos los documentos, datos, estadísticas, informes, programas de computadoras, llamadas telefónicas, etc., elaboradas por el gobierno, empresas particulares o personas físicas, es información que varía de acuerdo a su contenido, destino y proveedor en:

\section{a) Pública \\ b) Privada}

Esta división se analizará más adelante, debido a que el interés de este punto es exponer la importancia de la economía de la información.
En las sociedades industriales, el procesamiento de información "bruta" para ser convertida en información utilizable por un amplio número de usuarios, tiene un papel cada vez más importante. En efecto, en los diferentes sectores de la economía, un considerable número de personas está dedicado a actividades relacionadas de una u otra forma con la información.

Pasado el año de 1950, las industrias claves como la textil, la siderúrgica y la automotriz, entre otras, perdieron los primeros lugares en el ámbito económico y se vieron sustituidas cada vez más por industrias de nueva creación como son: la electrónica, la aeroespacial, la bioquímica, la biomédica, etc. Es en todas las últimas, donde la información tiene un papel de suma importancia.

Por otra parte, en las industrias donde la producción de servicios y productos es de mayor complejidad, la información adquiere un importante valor frente a las fuerzas de trabajo y materias primas.
Un gran porcentaje de los costos de producción industrial son enfocados hacia la información, como los relacionados con las investigaciones, desarrollo económico, explotación de mercados y publicidad. Además, la importación y exportación de los productos industrializados exige un mauro tráfico de información, con la finalidad de contactar a todas las unidades geográficamente dispersas de las trasnacionales por medio de elaboradas redes de información que coordinan el tráfico de los productos.

$\mathrm{El}$ sector servicios ha adquirido en nuestros días un papel relevante en la economía de la información, debido a que muchas de sus actividades (las telecomunicaciones, los transportes, la banca, etc.) están relacionadas con el procesamiento de la información. Por ejemplo, en el caso de México el Producto Nacional Bruto se divide en:

$\begin{array}{ccc}\text { Agricultura } & \text { Industria } & \text { Servicio }^{1} \\ \mathbf{1 0 \%} & 35 \% & 55 \%\end{array}$

\footnotetext{
* Jefe del departamento de Bibliotecas del Centro de Información Científica y Humanística de la UNAM.
} 
Esto es un buen parámetro para darnos una idea del gran apoyo que los países en vías de desarrollo le están dando al sector servicios, que se conjuga directamente con la economía de la información, debido a que el mayor porcentaje de servicios en el mundo se encuentra basado en el manejo constante de información, asimismo ofrecen grandes cantidades de este insumo.

Es bueno señalar que los países con un gran sector de servicios poseen una industria de la información altamente desarrollada. Esta gran industria, se constituye principalmente por las corporaciones que producen y distribuyen bienes y servicios de información como: la industria editorial, las productoras de computadoras y equipos de telecomunicaciones, las disqueras, la prensa, la radio y televisión, las empresas publicitarias, etc., y que están adquiriendo, con una rapidez sorprendente, una importancia de magnitudes considerables tanto en el plano nacional como internacional.

La información es actualmente un producto más en donde las grandes empresas trasnacionales tienen puestos sus ojos con la finalidad de obtener importantes ganancias económicas al: venderla, intercambiarla, producirla o almacenarla. De hecho ya se considera a nuestro tiempo como la "era de la información", debido a que este insumo es un punto fundamental para el buen desarrollo personal, institucional, regional o nacional, y es hacia donde una gran parte de recursos humanos y económicos se encuentran encaminados.

Por otra parte la información ya no sólo viaja por los canales tradicionales como libros y revistas, sino que llega a nuestros hogares por diversos medios electrónicos, inundando nuestra privacidad con anuncios, noticias, eventos deportivos, etc.

\section{Información y poder}

Desde hace algún tiempo se ha manejado que quien tiene información tiene poder. Esto es relativo, debido a que hacen falta una serie de condiciones para lograr que la información facilite el ejerció de un poder. Hace falta, para lograr ese poder, no sólo la materia prima de la informa- ción, sino también la infraesructura necesaria para su procesamiento y los contactos sociales para su utilización en la toma de decisiones.

Es una constante que la información se relacione con la toma de decisiones sociales, es por ello que resulta fundamental contar con capacidad analítica para determinar con exactitud cuál es la información apropiada, que se puede obtener mediante el análisis de cantidades masivas de información a través de medios electrónicos.

La información no sólo es en nuestros días un mecanismo de control social, sino también un producto que permite contar con un desarrollo económico que facilita al mismo tiempo un desenvolvimiento científico y tecnológico que producirá, en corto plazo, mayor cantidad de información que podrá ser comercializada a nivel internacional.

Es tal la idea de que la "información es poder" que un gran número de empresas se dedican a comercializar este nuevo recurso que rinde cada día mayores ganancias.

Esta comercialización se da a distintos niveles, desde la venta de productos de consumo de primera necesidad, hasta la competencia de los sistemas noticiosos del mundo, donde las cadenas televisivas buscan novedades y alternativas para que sus programas de noticias lleguen a un mayor número de personas, al igual que los diarios, las cadenas de radio y los programas de diversión y entretenimiento familiar.

Por otra parte, vemos a las grandes compañías dedicadas a monopolizar y comercializar bases de datos a nivel mundial de tipo referencial, texto completo, hipertexto, etc., con información de carácter científico como DIALOG y BRS o con datos de tipo general y recreativos como las promovidas por COMPUSERV.

A nivel mundial existen numerosos países productores de la información, pero son pocos aquellos que han tomado conciencia del poder que se puede obtener de ella, debido en gran parte a la falta de recursos tecnológicos para procesarla.
Actualmente los países de alto desarrollo tecnológico, económico, político y social son los que han determinado y marcado las pautas a seguir en el procesamiento industrial de la información.

\section{LA INFORMACIÓN COMO INDUSTRIA}

Como se ha visto anteriormente, la información (no como producto final) se puede considerar como: una materia prima, un producto sin acabado final, o un recurso físico que tiene ciertos atributos, tales como:

a) Valor, como las materias primas, la mano de obra o el dinero, el cual se puede tabular y controlar y al mismo tiempo abrir al mercado para su consideración.

b) Características cuentificables, en lo que respecta a su empleo, duración y efectos sobre otros recursos. Por tal motivo la información puede ser contada y combinada con otros factores como tiempo y cantidad usada.

c) La característica del control y presupuesto.

d) La posibilidad de relacionarse, en cuanto a sus costos y valores de uso, con los objetivos administrativos. ${ }^{2}$

El hablar de la información como: insumo, industria o economía, implica un costo en su producción, proceso y distribución en cualquier ámbito.

En la década de los 80, el aumento de la información así como los altos avances tecnológicos de las telecomunicaciones, han provocado un desarrollo de gran importancia dentro de la información. Gracias a ello, la industria de este "producto" ha alcanzado niveles superiores a los esperados y en nuestros días es una fuente primaria de ingresos para los países.

La información para ser comercializada requiere de una serie de elementos y procesos que la llevan a ser un producto vendible, entre los que se encuentran:

a) Su producción que, como su nombre lo indica, consta en la elaboración de información que permite a un grupo determinado de personas conocer 
ciertos datos sobre algún tema en particular.

La información producida puede ser de dos tipos: privada y pública, que a su vez se divide en: científica, técnica, comercial, bancaria, bursátil, industrial, humanística, general, noticieros, etc.

A esta información sin ningún tratamiento, sino como simple documento, dato, gráfica, etc., se le puede considerar como la materia prima par ala elaboración de la información de carácter comercial lucrativa o no lucrativa, en el complejo mundo de la información como industria.

b) Organización: por otra parte, la organización de la información para ser almacenada se considera uno de los puntos fundamentales en el valor económico, cuantitativo y cualitativo con que va a contar el producto al final del proceso.

La organización consistirá, en primer lugar, en la selección de la información que pueda ser útil para el fin deseado. Posteriormente se efectuará un acopio de lo seleccionado con el propósito de poseer la información.

El segundo paso de la organización consiste en clasificar a la información con base en su contenido, utilidad, finalidad, usuarios, servicios, etcétera, que se pretendan alcanzar con el proceso.

Esta etapa intermedia es de gran importancia debido a que para que la información como industria posea un valor económico debe ser de buena calidad para tener un mercado amplio. En este momento es donde se inicia el empleo de computadoras y telecomunicaciones para acopiar y clasificar la información, y es sin duda el uso de ambas herramientas lo que le darán un valor al producto en la economía de la información.

c) Almacenamiento: el almacenamiento de la información, organizada previamente, se efectúa por medios automatizados que agilizan todo tipo de proceso.
En esta parte los industriales de la información invierten enormes cantidades de presupuesto, que será recuperado posteriormente con a venta del producto terminado.

d) Distribución: esta es la última etapa del proceso de la información como industria y consiste en enviar la información al lugar que se requiera, sea local, regional, nacional o internacional, con base en una serie de necesidades de los consumidores y prestadores de servicios de información.

La distribución se efectúa empleando, en la mayoría de las ocasiones, los más altos avances tecnológicos relacionados con la computación y las telecomunicaciones, con el objetivo de agilizar al máximo en envío y recepción de información masiva o por paquetes a los lugares donde se requiera para su uso.

La venta de la información se da en todos los niveles y en todos los ámbitos de la vida, es por ello que la sociedad acepta esta actividad sin conocer todo el procedimiento por el que pasó para llegar a sus manos. Debido a esta situación algunos denominan a nuestra sociedad como la "sociedad informatizada".

Como se ha visto, la información como industria es una de las partes de la economía de la información que se compone de una materia prima (información en "bruto"), procesada técnicamente por medio de herramientas computacionales y distribuida a nivel internacional a través de modernas telecomunicaciones. Todo este proceso posee un costo y las personas y organizaciones que invierten en él procuran obtener al máximo una ganancia.

Sin embargo, independientemente de que el producto final (información) cuente con un precio, existen instituciones de carácter no lucrativo que toman a la información como un elemento que permite el desarrollo de su organización, estado, región o país. Aunque esto no quiere decir que la información no cueste, tiene un valor, pero éste no se nota, debido a que la mayoría de las veces se encuentra subsidiado por el gobierno a alguna otra institución.

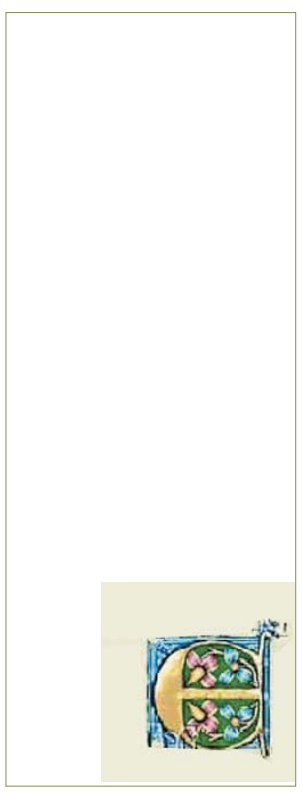

\section{La información como industria} es una de las partes de la economía de la información que se compone de una materia prima (información en "bruto"), procesada técnicamente por medio de herramientas computacionales y distribuida a nivel internacional a través de modernas telecomunicaciones. 
Por lo regular, los organismos no lucrativos son subsidiados por el gobierno de un país o instituciones de altos recursos económicos con fines científicos.

Un ejemplo clásico en México, es el Instituto Nacional de Estadística, Geografía e Informática (INEGI) quien produce una gama amplia de productos y servicios de información que en su mayoría son ofrecidos gratuitamente a otros organismos gubernamentales tanto nacionales como extranjeros y en un gran número de casos son vendidos a particulares a costos que varían (dependiendo del servicio), con el objeto de recuperar los gastos de producción del producto o servicio ofrecido y un porcentaje de ganancias para el mejor funcionamiento del organismo.

Actualmente la información como producto final en su industrialización, se puede considerar como un recurso de carácter:

a) Estatal o público: que facilita la operación de instituciones gubernamentales tanto en el territorio nacional como en el extranjero y al mismo tiempo agiliza la política nacional y su relación con otros países.

Por otro lado, la información se debe de considerar como un recurso nacional básico que debe ir a la par con la producción agrícola, energética, etc., por el simple hecho de que su acumulación, procesamiento y distribución permiten el adelanto científico-técnico del país.

Por lo que respecta a la normatividad de la información, no sólo los países desarrollados quienes han efectuado actividades relacionadas al tema, pero no se ha logrado crear hasta la fecha una política o una norma que permita controlar la información a nivel internacional. En el caso de México, los autores Reynel y Vélez ${ }^{3}$ proponen una serie de políticas e instrumentos para el desarrollo económico del "Sector Informacional" del país, como ellos lo nombran, donde las políticas a seguir las dividen en 14 grandes rubros que van desde la promoción de la alfabetización de la población mexicana, hasta la creación de "un ambiente legisla- tivo que propicie el desarrollo del Sector Informacional de la economía". 4

Los instrumentos propuestos, ligados a las políticas, cubren puntos como: la creación de organismos que apoyen, agrupen y fomenten a "los productoes y/o comercializadores de la información", la vinculación entre instituciones académicas con empresas (principalmente particulares); la elaboración de fuentes secundarias con base en colecciones documentales de información primaria; y la elaboración de programas de posgrado enfocados a la tecnología y ciencias de la información para una mejor formación de recursos humanos especializados que pueden ser estimulados por medio de becas. ${ }^{5}$

b) Individual: el recurso de la información deberá ser administrado de forma personal para que facilite al individuo un desarrollo profesional, social, económico, político, etc., y que le permita, al mismo tiempo, producir distintos tipos de información que a su vez, le sean útiles a otras personas, organizaciones, institutos del gobierno, etc.

c) Empresarial o industrial: la información previamente procesada permite a las industrias, organismos e instituciones lucrativas o de servicio social, llevar a cabo su organización interna, desde el aspecto administrativo hasta el tecnológico.

La información permite a las empresas optar por mejores decisiones, crear nuevos productos y servicios, mejorar la calidad de la producción, seleccionar tecnologías que apoyen el desarrollo industrial, conocer técnicas de producción de mayor calidad, etc. Por lo tanto la información permite un mejor funcionamiento interno y externo de la empresa.

La información se puede considerar como un bien desde el momento en que la buscamos para cubrir una necesidad. Pero no sólo se pretende cubrir una necesidad de información, sino que también se busca una satisfacción personal, que nos permita llevar a cabo actividades académicas, productivas y de control social, entre otras.
La información como bien no puede ser acaparada como un objeto propio, aunque algunos organismos pagan por su exclusividad o por su amplia difusión. Sin embargo, en el momento en que se obtiene (de forma gratuita o comprada) se pretende aprovechar al máximo su contenido.

En el momento en que se adquiere la información, también se obtiene un conjunto de características que satisfacen una serie de necesidades que se requieren cubrir, que van desde lo particular hasta lo más general. Es decir, si tenemos la necesidad de adquirir información sobre la automatización de bibliotecas en México para un trabajo de investigación y detectamos que se encuentran en una base de datos de texto completo que se localiza en una biblioteca especializada en computación, acudimos a ella para satisfacer nuestra necesidad.

La información recuperada satisface la necesidad de información requerida para nuestra investigación. Su impresión en hojas separadas con todos los datos de donde proviene la información, satisface en menor medida, el manejo y almacenamiento de la información en nuestra oficina u hogar.

Por otro lado, el hecho de que la información sea tratada como un bien no quiere decir que sea obligatorio que tenga un precio en el momento en que la recuperamos. Toda información (desde su punto de vista económico) tiene un costo de producción, pero cuando la adquirimos puede ser por pagar su precio total de elaboración u obtenida gratuitamente por una institución.

\section{Las Transnacionales de la Información}

En un estudio realizado por Hamelink en 1980, se detectó que son un total de 86 empresas trasnacionales las que controlan la producción y distribución de más del $75 \%$, aproximadamente, de los bienes y servicios de información a nivel mundial.

Los países dueños de estas industrias son: 51 Estados Unidos, 12 Reino Unido, 7 Japón, 5 Francia, 6 la República Federal Alemana, 1 Holanda, 1 Suecia, $1 \mathrm{Ca}$ nadá, 1 Italia y 1 compartida entre la República Federal Alemana y Holanda. 
De igual forma el mismo Hamelink divide a las empresas en tres amplias categorías:

1) Las de gran importancia a nivel internacional dentro de los conglomerados industriales con una participación activa en distintos sectores industriales, como: La Paramount Pictures, la Transamérica, y la Narner Communication, entre otras.

2) Las que forman parte de grupos empresariales que invierten su economía en diversos sectores de la industria de la información como son: la RCA, la Philips, etc.

3) Las empresas dedicadas a un sector específico de la industria de la información como: IBM, Erickson, Polygram, sony, etc.

Lo anterior, a su vez, se puede subdividir en:

a. Productores de bienes de capital (los productores y distribuidores de computadoras).

b. Productores de bienes intermedios (recopilación de datos como bases de datos).

c. Productores de bienes de consumo final (la información estadística, los discos, los noticieros, etc.).

Es bueno aclarar que las primeras empresas trasnacionales de la industria de la información fueron las agencias noticiosas, el teléfono, el telégrafo, el telex y posteriormente la industria de los hardware.

Sin embargo, para que la información sea trasnacionalizada por medio de grandes industrias requiere de:

a) Mayores tasas de crecimiento en un mercado interno.

b) Que la información produzca márgenes de utilidad más altos fuera del mercado interno.

c) Estímulo de las empresas productoras de bienes y servicios de información que están penetrando a nuevos mercados en el extranjero. d) Atracción del producto (información) para las empresas locales.

e) La extensión que se pretenda arriesgar financieramente en la trasnacionalización de la información.

Por último diremos que, día a día las empresas trasnacionales de la información cuentan con un desarrollo espectacular por la gama tan amplia de bienes y servicios de información que se ofrecen, gracias en parte al desarrollo tecnológico de las telecomunicaciones que faciliten el envío y recepción de datos.

\section{La Banca y la Información Financiera en la Información como Industria}

La información de carácter financiero circula a nivel mundial, en forma de datos de computadora, a través de redes bancarias y de las agencias noticiosas. Las redes son propiedad de los principales bancos transnacionales, que junto con las agencias de noticias, que también son de origen internacional, conforman los organismos más poderosos que se encuentran en excelentes condiciones de emplear la información que circula por canales más tradicionales.

De igual forma que sucede con el flujo de información de cualquier índole, existe, por una parte, un flujo de información financiera dentro de los países desarrollados y, por otro, un flujo entre los países desarrollados con los que están en vías de desarrollo. La información proporcionada por los países no desarrollados es información en "bruto", a diferencia de los países desarrollados que es información procesada.

A partir de los 60 existe un estrecho vínculo entre el sistema bancario y la industria de la información que constituye una prueba palpable de la relación de la economía con la información. Lo que provoca una desventaja a los países no desarrollados por su gran dependencia de información financiera de los países desarrollados, cuyas instituciones bancarias trasnacionales disponen de información al instante de cualquier índole monetario, gracias a las gigantescas redes de telecomunicaciones que conectan a todos los Bancos Centrales. Redes que carecen los países no desarrollados, por

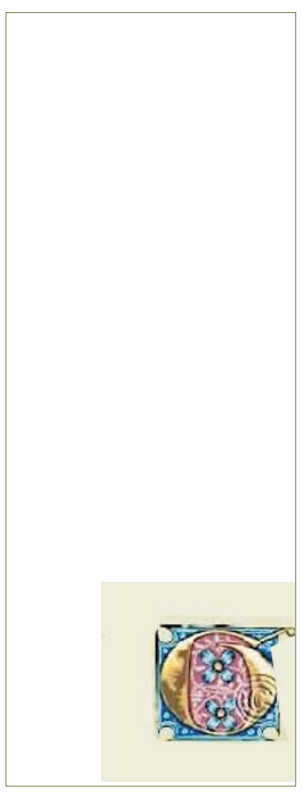

Toda información (desde su punto de vista económico) tiene un costo de producción, pero cuando la adquirimos puede ser por pagar su precio total de elaboración u obtenida gratuitamente por una institución. 
su alto costo y que por consecuencia crea una distancia cada vez mayor entre éstos y los países de primera línea.

\section{La información como industria en México}

México, país de gran importancia en américa Latina y uno de los primeros productores y consumidores de información cient $\{$ ifica, técnica, financiera, general, noticiosa, etc., cuenta, hasta el momento, con un importante complejo de telecomunicaciones que le permite enviar y recibir enormes cantidades de información de cualquier tipo.

Gracias al lanzamiento de los Satélites Morelos, nuestro país ha superado retos de comunicaciones de gran relevancia, que permiten a todos los centros de investigación, bibliotecas, centros de documentación, etc., el acceso a la información actualizada que se localiza en bancos y bases de datos en todo el mundo, con un mínimo de problemas para su contactación.

La información en México, es aceptada como un fenómeno natural que debe fluir por cualquier medio, sea este científico, comercial, bancario, etc. Pero no es considerada como una fuente primaria de ingresos, ni como una materia prima que se debe procesar para su comercialización, sino como un producto del cual se cubran una serie de necesidades de cualquier índole, no importando como se produzca.

Hasta la fecha son pocas las instituciones en México, que se dedican a procesar la información para comercializarla. Todavía no se toma una plena conciencia del gran valor político, social, económico, intelectual, etc., que da la información recuperada, procesada, almacenada y difundida por medios electrónicos.

Por lo tanto la información como industria no ha alcanzado una lto rendimiento en el país, pero a la fecha organismos como el INEGI (Instituto Nacional de Estadística, Geografía e Informática) o la UNAM (Universidad Nacional Autónoma de México) poseen una infraestructura tal que permite generar enormes cantidades de información científico-técnica y que a su vez es preparada y almacenada en medios electrónicos que facilitan su recuperación. Pero su comercialización no ha cubierto rangos de importancia por ser organismos de carácter gubernamental que subsidian los gastos de producción, ofreciendo, en muchos casos, la información de manera gratuita.

Por otro lado, tenemos a la Universidad de Colima, primera universidad del país con capacidad para producir CD-ROM, quien presta sus servicios a otras instituciones para almacenar su información en CD-ROM a un costo mínimo, por el hecho de ser un organismo estatal subsidiado por el gobierno, y distribuye el fruto de este almacenamiento de información de manera gratuita a diversas instituciones gubernamentales bajo un convenio.

Es bueno aclarar que, en el momento en que hablamos de la información como industria es importante resaltar:

1) Para que la información pase a ser industria se requiere (como se analizó anteriormente) de medios electromagnéticos, así como de sistemas de telecomunicaciones. Por lo tanto para que la información sea procesada y transmitida se debe efectuar una inversión económica, cubierta por organismos privados o gubernamentales, con base en los objetivos que pretendan alcanzar con la información procesada.

2) Se requiere de organismos gubernamentales o particulares que financien su producción, recopilación, proceso, almacenamiento y distribución.

3) Debe existir un mercado donde pueda ser introducida la información como un bien o servicio.

Por otra parte, la información en nuestro país es subsidiada en un alto porcentaje y por lo tanto pasa a ser una industria no lucrativa.

\section{La información no lucrativa}

En casi todos los países existen dos tipos de información, que son: a) La información vendible o comercial y b) La información no lucrativa.
Ahora trataremos lo que respecta a la información no lubrativa.

El ser humano tiene una tendencia "caritativa" que le permite apoyar a personas, productores, servicios, bienes, etc., con base en sus necesidades, recursos, intereses, etc.

En casi todo el mundo, las personas u organismos donantes de economía o servicios pretenden subsidiar a instituciones del gobierno con fines sociales.

En México son las instituciones gubernamentales junto con organismos particulares de carácter "filantrópico" quienes se encargan de subsidiar una enorme cantidad de bienes y servicios de información.

Por lo tanto, la información producida por los organismos de gobierno subsidiados por otras instancias es por lo regular información que se ofrece a bajo costo y en casos especiales se "regala".

Pero no necesariamente la información necesita ser subsidiada por instituciones particulares, también el mismo gobierno subsidia un gran número de bienes y servicio de información, desde los inicios de la información educativa de los niños (con el libro de texto gratuito), hasta la consulta a bancos de información a nivel mundial que realizan los investigadores, académicos y en alguns casos alumnos de universidades estatales.

Dentro de la información como industria, el caso de la producción de la información es subsidiada como producto de consumo final, en gran medida con el objetivo de que tenga acceso por un gran número de usuarios, provocando con ello un desarrollo económico, político, social y educativo del país.

La información no lucrativa pretende servir a una comunidad heterogénea que procura recuperar información para cubrir una serie de necesidades que la llegan a consumir enormes cantidades de información que no podría pagar en un momento dado. Es por ello que el subsidio a la información en México, es y será una constante, con el propósito de avanzar cada vez más social, política y económicamente, por el simple hecho de que, si se ofrece información por medio de bienes y servicios el usuario de ésta generará nueva informa- 
ción que auxiliará en la producción de mayor cantidad de este bien.

\section{Las bases de datos en México}

En nuestro días, para que la información como industria sea tal, es necesario realizar una serie de procedimientos por medio de cuatro elementos: recursos humanos, software, computadoras y telecomunicaciones.

Estos elementos son característicos de las bases de datos, tanto las que se producen a nivel mundial como las que se elaboran en el Territorio Nacional.

Aunque en México ya existen bases de datos en disco compacto (CD-ROM) con información nacional ( $\mathrm{CICH}$, LIBRUNSAM, DIALES, etc.), se requiere de alta tecnología y personal capacitado para el procesamiento, almacenamiento y recuperación de información.

En el país no existe a la fecha un sistema único de Bancos de Información, pero esto no quiere decir que la producción de ellos se detenga.

El acceso a las bases de datos de cualquier tipo (Nacionales o Internacionales) tiene un valor económico que va desde el costo de las telecomunicaciones (en el caso en-línea) hasta la impresión de datos recuperados.

Muchas instituciones con el fin de que el país cuente con un mayor desarrollo científico-técnico subsidian los gastos de consulta de las bases de datos a sus investigadores. Pero como lo hemos mencionado anteriormente, todo esto tiene un costo, desde la producción de la información hasta que ésta llega a nuestras manos.

Las bases de datos desarrolladas en México y consultadas aquí mismo tienen un costo menor y si son instituciones no lucrativas tienen un costo aún menor para quienes tienen su acceso.

Las Bases de Datos de mayor importancia producidas en el país y que se pueden acceder por SECOBI son: ${ }^{8}$

a) Bases de datos actualizadas:

Aries

Biblat

Clase
Desa

Periódica

Sie-Banxico

UNAM-Jure

b) Bases de datos no actualizadas:

Banapa

Bive

Bidesre

Campa

Catb

Cyd

CCPS

Frosur

Lime

Mecs

Norm

Sife

Esto no quiere decir que las otras bases de datos existentes en México tengan menor importancia, sino que no son tan difundidas como las mencionadas.

Las bases de datos son un buen parámetro para determinar el desarrollo de la información en un país, por los pasos que emplea para entregar productos y servicios de información.

Es por ello que los países altamente desarrollados cuentan con una enorme industria de la información, que muchas ocasiones apoyan en gran medida al Producto Interno Bruto. ${ }^{9}$

\section{La Venta de Información en México}

Actualmente en nuestro país existen instituciones que se dedican a vender información con fines lucrativos. Ello es una pauta que permite vislumbrar poco a poco el interés de compañías particulares y de gobierno sobre la información como una industria para su comercialización.

Entre las compañías sobresalientes tenemos a INFOTEC, quien se encarga de recuperar información, en cualquiera de sus presentaciones, almacenarla y venderla a los clientes que lo soliciten a un precio determinado, tomando en cuenta

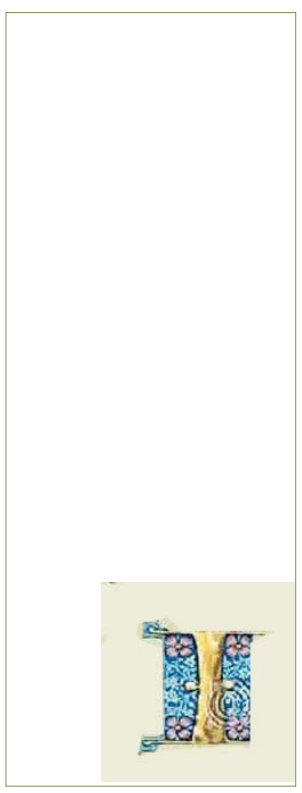

Para que la información como industria sea tal, es necesario realizar una serie de procedimientos por medio de cuatro elementos: recursos humanos, software, computadoras $y$ telecomunicaciones. 
el costo de la información más la ganancia de INFOTEC, más la inversión en las telecomunicaciones si es que se emplean. Por lo regular el precio pagado por la información es considerable, por tal motivo los clientes son instituciones particulares o gubernamentales que tienen la posibilidad de desembolsar altas sumas de dinero por ella.

Asimismo INFOTEC ofrece una gama amplia de servicios a empresas e industrias, entre los que se encuentran:

a) Servicios de Información:

Proporciona información técnica, científica, de mercado y financiera, incluyendo el Centro de Información sobre Comercio Exterior de BANCOMEXT.

\section{Ofrece además:}

Boletines mensuales de alerta, servicios telefónicos de consulta industrial, normas, patentes, directorios, perfiles de plantas industriales, fracciones arancelarias, catálogos de proveedores y equipo, estadísticas, indicadores económicos, índices, revistas, bancos de datos internacionales y recuperación de datos.

b) Servicios de Consultoría:

Ofrece consultoría en proyectos de Reconversión industrial, análisis de posición competitiva, desarrollo tecnológico de productos y procesos, transferencia de tecnología, evaluación de proyectos industriales, perfiles técnico-financiero. Y en el área de recursos humanos. Desarrollo organizacional y sistemas de sueldos y compensaciones.

c) Servicios de Capacitación:

Ofrece este servicio a las empresas e industrias que así lo soliciten para su personal ejecutivo. ${ }^{10}$

Otra compañía de servicios semejantes es Asesores Especializados en Información y Documentación., S. C., (AEIDOC), compañía encargada en recuperación de información en base de datos y obtención de documentos primarios a nivel mundial. La información la obtienen de bases como ERIC, BIOSIS, CAB, LISA, etc., y los documentos pueden ser tesis, artículos de revistas, patentes, etc.
Sus costos son variados y fluctúan entre los 20 (obtención de artículos) y 150 dólares (información sobre patentes). ${ }^{11}$

Por otro lado, tenemos a la compañía norteña INFOSEL (Información Selectiva) que la conforma un área del periódico "El Norte" del estado de Nuevo León, y que ofrece servicios sobre información económica, así como su servicio de Infosel Financiero, que es trasmitido por satélite desde Nuevo León e incluye información diaria del mercado de valores, de la bolsa de valores, del dólar y su cotización en distintas partes del mundo, etc. El servicio tiene un costo, en 1991, de 595 dls. mensuales más $150 \mathrm{dls,} \mathrm{por} \mathrm{compu-}$ tadora adicionalconectada.

Otras instituciones que se encargan de vender información de tipo financiera en México son:

a) El Centro de Información de la Bolsa Mexicana de Valores, que por medio de sus diversas áreas mantiene actualizados a los interesados en información sobre cuestiones bursátiles y financieras de empresas, así como de sus valores. ${ }^{12}$

b) El Centro de Información NAFIN, quien se encarga de difundir la información, primero de manera interna y luego eterna, sobre aspectos técnicos de las diversas áreas de NAFIN y otros relacionados con las finanzas.

c) El Centro de Información Financiera y Económica del Instituto Tecnológico de Estudios Superiores de Monterrey (ITESM), quien se encarga de apoyar al sector empresarial, de investigación y académico del país con el fin de agilizar la toma de decisiones, "dentro de un ambiente de competitividad internacional en las áreas financiera, económica y afines". 13

Como se ve son pocas las industrias que se encargan de considerar a la información como un producto que se puede comercializar, además todas son de carácter privado.
Es indispensable que las instituciones de carácter público en el país, tomen en cuenta este tipo de servicios para su comercialización puesto que ellos son generadoras de enormes volúmenes de información que le pueden ser útiles a un gran número de usuarios, no sólo a nivel nacional sino también internacional.

\section{La información científica y tecnológi- ca en México (Cifras 1990) ${ }^{14}$}

La formación de recursos humanos de alta calidad y productividad, el desarrollo científico y la modernización tecnológica, son elementos fundamentales para que México cubra sus objetivos de bienestar y desarrollo par ala totalidad de sus habitantes y logre con ello ina inserción ventajosa en los mercados internacionales.

Tomando como base la gran importancia con que cuentan estos aspectos dentro de la política de desarrollo económico del actual gobierno mexicano, es indispensable resaltar los indicadores básicos relacionados con la tecnología, ciencia, transferencia de tecnología y propiedad industrial en un tema como lo es la información como industria.

Cabe señalar que en general la información sobre ciencia y tecnología es escasa y aún no existen mecanismos adecuados que concentren esta información satisfactoriamente y den amplia cobertura, principalmente lo referente al desgloce de gastos e inversiones en ciencia y tecnología del sector privado.

El 9 de enero de 1990 se publicó el nuevo "Reglamento de la Ley sobre Control y Registro de la Transferencia de Tecnología y el uso y Explotación de Patentes y Marcas". Un mes después (febrero de 1990) se dio a conocer el "Programa Nacional de Ciencia y Modernización Tecnológica, 1990-1994". Ambos documentos son parte importante de la información como industria, ya que permiten regular la formación de recursos humanos, en particular en la actividad científica del país, para que camine a la par con las corrientes mundiales del conocimiento y así apoyar la detección de los problemas nacionales en las diversas áreas del desarrollo científico y tecnológico. 
A continuación analizaremos las cifras relacionadas con los indicadores sobre tecnología.

\section{Cifras 1990}

Dentro de la información como industria y la economía de la información, las telecomunicaciones forman parte indispensable. En México se efectúa una inversión importante en ellas y cada año se incrementa esta cantidad para su desarro1lo. El consejo Nacional de Ciencia y Tecnología (CONACYT), dividió el gasto público en ciencia y tecnología en diez sectores, donde las telecomunicaciones nunca han estado dentro de los tres primeros lugares /véase cuadro 1).

Por lo que respecta al gasto total de CONACYT, de 32 conceptos hacia donde enfoca su economía, 6 se dedican al desarrollo y difusión de información científica-técnica, de los cuales ninguno ocupa uno de los 4 primeros lugares (véase cuadro 2).

Por otro lado, los programas que apoyó el CONACYT en los años 1983-1987 son 17, pero sólo 4 de ellos son programas dedicados al desarrollo y difusión de la información científica-técnica del país (véase cuadro 3).

Los proyectos apoyados por el CONACYT durante 1985-1989 se dividen en un total de 16 conceptos y sólo 4 de ellos son los encargados en llevar a cabo el desarrollo, difusión y reglamentación de la información (véase cuadro 4).

Como se puede ver, son pocos los proyectos enfocados propiamente al desarrollo de la información que CONACYT apoyó durante este periodo, se hace notar que en dos de los conceptos nunca se realizó investigación (véase cuadro 4).

Por otra parte la Universidad Nacional Autónoma de México (UNAM), la Secretaría de Educación Pública (SEP) y la Asociación Nacional de Universidades e Instituciones de Educación Superior (ANUIES) también ofrecen apoyo a diversos proyectos de desarrollo de información en el país, entre los que destacan:

a) La Red de Cómputo de la UNAM (REDUNAM), que une por medio de telecomunicaciones a todas las depen- dencias de nuestra máxima Casa de Estudios.

b) La Red de Telecomunicaciones dela SEP, cuyo objetivo es unir a las universidades públicas del país por medios electrónicos, denominada RUTIC (Red Universitaria de Teleinformática y Comunicaciones).

c) La Red de Bibliotecas Públicas del país, RENABIP (Red Nacional de Bibliotecas Públicas).

Como se mencionaba al principio del trabajo, es de gran importancia conocer datos de este tipo para detectar el desarrollo de la información como industria en México, que si bien cuenta con un apoyo y un desenvolvimiento paulatino, la información sobre el tema es escasa.

Cabe señalar que, si esta información se tomara en cuenta como un parámetro para medir el desarrollo de la información en el país, se detectaría que del producto interno bruto de México una mínima parte proviene de esta industria de la información.

\section{La biblioteca y la información como industria}

Hace algunos años el mercado se encontraba enfocado y saturado de productos tangibles, que podrían ser manipulados y vendidos en distintos niveles. Hoy en día los servicios, (de cualquier índole) están tomando tanta o más importancia que estos productos y los países enfocan una enorme cantidad de divisas a esta nueva economía.

Las bibliotecas (y demás sinónimos), son organismos encargados de ofrecer servicios a una comunidad determinada, por lo tanto encajan dentro de esta nueva industria.

La información localizada y ofrecida dentro de las bibliotecas del mundo, es en su mayoría gratuita, pero en la actualidad existen organismos que cobran ciertos servicios de información especializados. Sin embargo, todavía las bibliotecas en los países en vías de desarrollo juegan un papel poco relevante como unidades de negocios en la industria de la información.

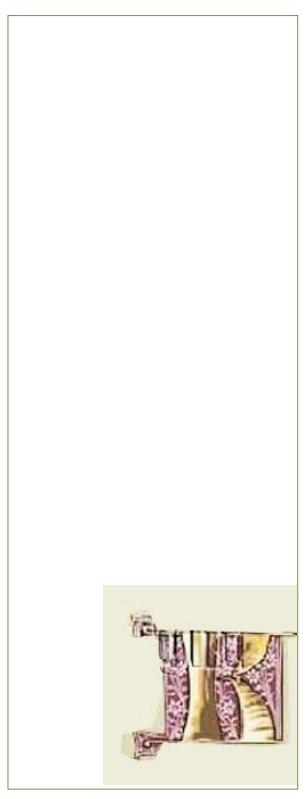

La información localizada y
ofrecida dentro de las bibliotecas del mundo, es en su mayoría gratuita, pero en la actualidad existen organismos que cobran ciertos servicios de información especializados. 
La asociación de la mercadotecnia y la información, es un fenómeno natural en las empresas dedicadas a ofrecer servicios de consultoría, distribución de la información, creación de bases de datos, elaboración de publicaciones, etc., y asumen estas actividades como negocio, con mayores o menores márgenes de utilidad. Pero desafortunadamente este punto de vista de la información no se ha trasladado a nuestro propio contexto en la biblioteca.

Al respecto, la autora Carrión menciona que: "El campo de la información, el de nuestra especialidad, en el que hemos desarrollado competencias y habilidades, no lo hemos abordado, con un enfoque de negocio, con una mente empresarial." 15

Desde el punto de vista del bibliotecólogo, resulta paradójico, debido a que nos quejamos de los elevados costos de adquisición de productos y servicios de información, pero no actuamos enérgicamente con el objeto de buscar caminos y alternativas que nos permitan generar ingresos propios.

Debemos de tomar en cuenta, como bibliotecólogos, que por lo que respecta a la información científico-técnica que se localiza en las bibliotecas, centros de documentación, etc., podemos obtener ingresos al crear servicios de información de mayor calidad y sofisticación.

Es indispensable evaluar y analizar si los productos y servicios de información que ofrece nuestro organismo responden a necesidades claras del mercado potencial de nuestros usuarios, pues de no ser así, se tiene que tomar la decisión que hasta la fecha se lleva en las bibliotecas: suspender todo tipo de venta de información que reditúe fondos.

En la biblioteca como en cualquier otra empresa o institución el conocimiento del comportamiento de nuestros futuros compradores y distribuidores es relevante para que la oferta de información se ajuste a ellos, por eso se toma como pará- metro de efectividad de los servicios, la satisfacción de las necesidades de información "de los clientes" (usuarios).

No cabe duda que el profesionista de la información (bibliotecólogo) debe estar preparado siempre para manejarse dentro del mundo de los negocios, donde existe una enorme competencia.

Para que la biblioteca y el bibliotecólogo decidan establecer servicios de información "vendibles o comerciales" deben de llevar a cabo una cuidadosa planeación estratégica, la que iniciaría con el reconocimiento de los servicios y recursos con que cuentan, así como las necesidades que se piensa satisfacer del usuario. Asimismo se detectarán los objetivos que se piensa cubrir, los pros y los contras del servicio; dónde y cuando se llevará el servicio; la disponibilidad de los recursos económicos, materiales y humanos; y el impacto que se espera obtener en este mercado.

Si el bibliotecólogo ve a su institución no solamente como una prestadora de servicios, sino como una fuente de información "comercial", es preciso que contemple los puntos anteriores para que funcione mejor su proyecto de venta de información. Y aún más, debe de considerar situaciones tales como: la persona encargada de ofrecer los productos y servicios de información y que sirve de intermediario entre estos y el "cliente"; los medios de comunicación que se emplearán para la distribución de la información; y el precio que se determine al servicio o producto en el momento en que se entrega al usuario.

La fijación del precio de los productos y servicios de información no es una tarea fácil, porque se debe de considerar la calidad del servicio/producto; las horas-hombre gastadas en su producción; el profesionalismo y capacidad del personal asignado a la labor; y los medios tecnológicos empleados, entre otras cosas.
Por otra parte el profesional de la información (bibliotecólogo), siempre debe de tomar en cuenta, para vender información, que ésta es un insumo indispensable para el desarrollo de los sectores industrial, académico, económico, político y de investigación, y que para su producción se requieren altos egresos. Porque sin duda alguna, los productos y servicios de información poseen un potencial para generar ingresos en beneficio de la biblioteca que los brinde.

Ejemplos tangibles de bibliotecas y centros de información que obtienen importantes ingresos en la venta de información en México son las ubicadas en las siguientes instituciones: la Bolsa Mexicana de Valores; el Centro de Información Científica y Humanística; el Instituto de Investigaciones Eléctricas; la Secretaría de Comercio y Fomento Industrial; el Banco Nacional de México, etc.

Aquí es donde se debe tomar muy en cuenta que la inversión en servicios y productos de información reditúe economía a la institución e incremente a su vez la productividad. Por otra parte, se debe de considerar el costo y el tiempo invertidos en la recuperación de información para valorar si cubre nuestras necesidades, pero esto no quiere decir que la información obtenida sea buena. Para evaluarla se debe de tomar en cuenta la información misma, de otra manera se caerá dentro de los sistemas de administración de mala información.

En México es el momento, como uno de los principales países productores de información de América Latina, para que se dé un nuevo enfoque a la carrera de bibliotecología, con el fin de entrar en el amplio mercado de la información "comercial", debido a que lo están acaparando instituciones dedicadas a este propósito, pero que no cuentan con la riquísima información existente en nuestras bibliotecas.

\section{NOTAS}

1 Datos proporcionados en los Indicadores del Desarrollo del Banco Mundial: 1976-1980.

2 VILLANUEVA Lara, J., "Información un recurso nuevo”, p. 18. 
3 REYNEL I., H. "El sector de la información en México", en Información: producción, comunicación y servicios 1 (3), May.-Jun- 1991, pp. 410.

4 Idem. pp. 8-9.

5 Idem. pp. $9-10$

6 HAMELINK, Cees., Finanzas e Información, México, ILET, 1984. pp. 38-43.

7 Idem.

8 CONACyT, Catálogos de Bancos de Información, México, 1990. México, el consejo, 1990. pp 2 у 3.

9 Véase:

"Electronic database revenues expected to reach $\$ 7.5$ billion in 89 ", en IDP Report; information and data base publishing report. 9(23), enero 20, 1989, pp. 1, 4-6.

"Proffessional database revenues represent $15 \%-20 \%$ of total on line business", en IDP Report; information and data base publishing report, 10(1), febrero 17, 1989, pp. 3-4.

10 INFOTEC. México, INFOTEC, 1991. 10 hojas desprendibles (folleto de propaganda).

11 AEIDOC: Asesores Especializados en Información y Documentación, S. C. México, 1992, 1 hoja (hoja de propaganda).

12 RAMÍREZ Escárcega, Aníbal y J. A. Yánez de la peña, "El centro de información de la Bolsa Mexicana de Valores", en: Información: producción, comunicación y servicios, año 2(3), 1992, pp. 30-32.

13 Centros de información financiera: BMV, NAFINSA e ITEMS", en Información: producción, comunicación y servicios, año 2(3), 1992, pp. 29-30.

14 Datos tomados de Nacional Financiera, La economía Mexicana en cifras 1990, México, NAFINSA, 1990, pp. 641-658.

15 CARRIÓN, Guadalupe, "Mercadotecnia de la Información”, en Simposio de sistemas de información, México, Instituto Mexicano del Petróleo, 1991, p. 80.

\section{BIBLIOGRAFÍA CONSULTADA}

AEIDOC: Asesores Especializados en Información y Documentación, s. C., México, 1992, 1 hoja (hoja de propaganda).

AMAT, NURIA, La biblioteca electrónica. Madrid, Fundación Germán Sánchez Ruipérez, 1990. (Biblioteca del libro), 206 p.

CARRIÓN, GUADALUPE, "Mercadotecnica de la información”, en Simposio de sistemas de información. México (Ciudad) 1990. Memoria, México, Instituto Mexicano del Petróleo, 1991, pp. 78-91.

"Centros de información financiera: BMV, NAFINSA e ITEMS", en Información: producción, comunicación y servicios, año 2(3), 1992, pp. 29-30.

CONSEJO NACIONAL DE CIENCIA Y TECNOLOGÍA, Catálogo de bancos de informaión, México, el Consejo, 1990, 183 p. 
"Electronic database revenues expected to reach $\$ 7.5$ billion in 89", en IDP Report; information and data base publishing report, 9 (23), enero 20, 1989, pp. 1,4-6.

Hacia el año 2000 ¿qué profesional de la información necesitamos en México? Rosa Ma. Fernández de Zamora [et. al.] México, Universidad Nacional Autónoma de México, Facultad de Filosofía y Letras, 1990, 58 p.

HAMELINK, CEES, Finanzas e información; un estudio de intereses convergentes, México, Instituto Latinoamericano de Estudios Trasnacionales, Nueva Imagen, 1984, $217 \mathrm{p}$.

"Indicadores sobre Tecnología"., en Nacional Financiera, La economía mexicana en cifras 1990, 11 a . ed., México, Nacional Financiera, 1990, pp. 641-658.

INFOTEC, México, INFOTEC, 1991, 10 hojas desprendibles (folleto de propaganda).

KUHLMANN, FEDERICO, Comunicaciones; pasado y futuro, México, Secretaría de Comunicaciones y Transportes, F. C. E., 1989, 257 p.

MADEC, ALAIN, El mercado internacional de la información: los flujos transfronteras de informaciones de datos. Madrid, FUNDESCO, Tecnos, 1984, $159 \mathrm{p}$.

Principles and aplications of information science for library profesionals, John N. Olsgaard, ed. Chicago, American Library Association, 1989, 142 p.

"Professional database revenues represent 15\%-20\% of total on line business", en IDP Report; Information on data base publishing report, 10(1), febrero 17, 1989, pp-3-4.

RAMÍREZ ESCÁRCEGA, ANIBAL y J. A. YÁNEZ DE LA PEÑA, “’”El centro de información de la Bolsa Mexicana de Valores”, en Información: producción, comunicación y servicios. 1(3), May-Jun. 1991, p. 4-10.

REYNEL IGLESIAS, HEBERTO y C. VELEZ SALAS, "El sector de la información en México: políticas e instrumentos para su desarrollo económico", en Información, producción, comunicación y servicios, 1(3), May-Jun., 1991, pp. 4-10.

"La sociedad de la información", en Información: producción, comunicación y servicios, México, año 1(1), enero-febrero, 1991, pp. 5-10.

STIGLER, G. J ., "la economía de la información”, en Economía de la Información y del conocimiento, Selección de D. M. Lamberton, México, Fondo de Cultura Económica, 1977, (Lecturas; 20), pp. 59-80.

TULLOCK, G. "Información no lucrativa”, en Economía de la información y del conocimiento, Selección de D. M. Lamberton, México, Fondo de Cultura Económica, 199, (Lecturas; 20), pp. 115-133.

VILLANUEVA LARA, JULIO, "Información: un recurso nuevo", en Boletín del Sistema Nacional de Información Científica y Tecnológica, 7(2) Jul.-Dic., 1988, pp. 18-20. 


\section{ANEXO 1}

Cuadro 1. Información proporcionada por el CONACYT sobre el gasto público en telecomunicaciones desde 1975 hasta 1988.

\begin{tabular}{|c|c|c|c|}
\hline Sector & Año & Lugar & Gasto Público \\
\hline $\begin{array}{l}\text { Comunicaciones y } \\
\text { transportes }\end{array}$ & $\begin{array}{l}1975 \\
1976 \\
1877 \\
1978 \\
1979 \\
1980 \\
1981 \\
1982 \\
1983 \\
1984 \\
1985 \\
1986 \\
1987 \\
1988\end{array}$ & 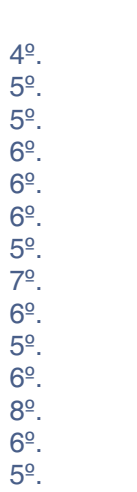 & $\begin{array}{l}\text { (Millones de pesos) } \\
189 \\
222 \\
293 \\
41 \\
114 \\
62 \\
693 \\
697 \\
3361 \\
5465 \\
3938 \\
2222 \\
19049 \\
2292\end{array}$ \\
\hline
\end{tabular}

\section{ANEXO 2}

Cuadro 2. Gasto total del CONACYT en apoyo a proyectos de investigación y difusión de la información.

\begin{tabular}{|c|c|c|c|c|c|}
\hline \multicolumn{6}{|c|}{ Gasto por año (millones de pesos) } \\
\hline Concepto & 85 & 86 & 87 & 88 & 89 \\
\hline Centros de & 27.7 & 40.5 & 1355.9 & 399.1 & 360.0 \\
\hline aoyado & 499.4 & 1090.2 & 983.7 & 1247.7 & 1131.5 \\
\hline $\begin{array}{l}\text { Tiraje de revistas } \\
\text { Libros } \\
\text { editados }\end{array}$ & 56.6 & 121.1 & 871. & 1381.7 & 1257.2 \\
\hline $\begin{array}{l}\text { Folletos } \\
\text { Informativos } \\
\text { publicados }\end{array}$ & 143 & - & 12.1 & 1652.3 & 1500.3 \\
\hline $\begin{array}{l}\text { Emisiones de } \\
\text { radio y T.V. }\end{array}$ & 148.8 & 240.8 & 559.2 & 52.88 & 486.1 \\
\hline
\end{tabular}

(-) Indica que no existió apoyo económico que no se llevaron a cabo actividades durante esos años. 
ANEXO 3

Cuadro 3. Gasto del CONACYT por año en apoyo a programas dedicados al desarrollo y difusión de información científico-técnica

\begin{tabular}{|l|c|c|c|c|c||}
\hline \multicolumn{5}{|c||}{ Gastos en apoyo a programas } \\
\hline \multicolumn{1}{|c|}{ Programa } & $\mathbf{8 3}$ & $\mathbf{8 4}$ & $\mathbf{8 5}$ & $\mathbf{8 6}$ & $\mathbf{8 7}$ \\
\hline $\begin{array}{l}\text { Desarrollo de servicios de información científica } \\
\text { y tecnológica }\end{array}$ & 133.3 & 339.2 & 368.9 & 703.6 & 1512 \\
$\begin{array}{l}\text { Difusión y divulgación de informática y } \\
\text { computación } \\
\begin{array}{l}\text { Regulación de la transferencia de tecnología } \\
\text { internacional }\end{array}\end{array}$ & 153.7 & 474 & 765 & 1480.6 & 2733.6 \\
\hline
\end{tabular}

(-) Indica que no existió apoyo económico que no se llevaron a cabo actividades durante esos años.

\section{ANEXO 4}

Cuadro 4. Proyectos apoyados por el CONACYT durante 1985-1989 dedicados al desarrollo,difusión y reglamentación de la información

\begin{tabular}{|c|c|c|c|c|c|}
\hline \multicolumn{6}{|c|}{ Número de proyectos } \\
\hline Concepto/año & 85 & 86 & 87 & 88 & 89 \\
\hline $\begin{array}{l}\text { Fomento para el desarrollo de servicio de } \\
\text { información científico-técnica }\end{array}$ & 13 & 18 & 28 & 14 & 14 \\
\hline $\begin{array}{l}\text { Fomento a la difusión y divulgación } \\
\text { científico-técnica }\end{array}$ & 12 & 1 & - & - & - \\
\hline $\begin{array}{l}\text { Regulación de la transferencia internacional de } \\
\text { tecnología }\end{array}$ & - & - & - & - & - \\
\hline
\end{tabular}

(-) Indica que no existió apoyo económico que no se llevaron a cabo actividades durante esos años. 